\title{
Effects of X-ray on the metacestodes of Echinococcus granulosus in vitro
}

\author{
Rui Mao ${ }^{1 \dagger}, \mathrm{Ge} \mathrm{Wu}^{1 \dagger}$, Hui Wang ${ }^{2}$, Pengfei $\mathrm{Lu}^{1}$, Jun $\mathrm{Li}^{2}$, Haitao Li², Aimudula Ainiwaer ${ }^{1}$, Yiwei Bai ${ }^{1}$, Mingyang Shu', \\ Yongxing $\mathrm{Bao}^{1 *}$ and Wenbao Zhang ${ }^{2^{*}}$
}

\begin{abstract}
Background: Radiotherapy may represent an alternative treatment modality for cystic echinococcosis (CE), but there is no adequate evidence for it up to now. In this study, we aim to investigate the parasiticidal effects of X-ray on the metacestodes of Echinococcus granulosus in vitro.

Methods: Protoscoleces obtained from sheep naturally infected with CE were cultivated in RPMI 1640 medium containing $10 \%$ fetal bovine serum (FBS) at $37{ }^{\circ} \mathrm{C}$ in $5 \% \mathrm{CO}_{2}$. Upon encystation on day 14 , the metacestodes were subjected to various intensities of $X$-ray. Metacestode structures were observed using light microscope and transmission electron microscopy (TEM), and Real-Time PCR was carried out to determine the expression of EgTPX, EgHSP70, EgEPC1 and Caspase-3.
\end{abstract}

Results: On day 14, encystation was noticed in the majority of protoscoleces in the control group. In the X-ray groups, the encystation rate showed significant decrease compared with that of the control group $(P<0.05)$, especially the groups subjected to a dose of $\geq 40$ Gy $(P<0.01)$. Light microscope findings indicated the hooklets on the rostellum were deranged in the irradiation group, and malformation was noticed in the suckers in a dose dependent manner. For the TEM findings, the cellular structure of the germinal layer of the cysts was completely interrupted by X-ray on day 7. The expression of EgTPX, EgHSP70, EgEPC1 and Caspase-3 was up-regulated after irradiation, especially at a dose of $\geq 45 G y(P<0.05)$.

Conclusions: X-ray showed parasiticidal effects on the metacestodes of $E$. granulosus. Irradiation triggered increased expression of EgTPX, EgHSP70, EgEPC1 and Caspase-3.

Keywords: X-ray, Echinococcus granulosus, Metacestode, Infection

\section{Background}

Cystic echinococcosis (CE), a chronic and neglected disease with liver as the most frequent location of parasitic cysts [1], is a zoonosis induced by the larval stage of the cestode Echinococcus granulosus. The liver is the most frequent location of echinococcal cysts, accounting for about $70 \%$ of the cases, and the lungs are the second most common location [2]. However, the cysts can be detected anywhere in the whole body, and the clinical symptoms of the hydatid disease are highly depending

\footnotetext{
*Correspondence: xydrui@sina.com; wenbaozhang2013@163.com

${ }^{\dagger}$ Equal contributors

'Department of Radiation Oncology, The First Affiliated Hospital of Xinjiang Medical University, Urumqi 830054, China

${ }^{2}$ State Key Laboratory Incubation Base for Xinjiang Major Diseases Research and Xinjiang Key Laboratory of Echinococcosis, The First Affiliated Hospital of Xinjiang Medical University, Urumqi 830054, China
}

on the size and sites of the lesions [3]. Surgery is preferred for treatment of CE. Nevertheless, severe anaphylactic shock may present in some patients due to postoperative release of fluid from the cysts. Besides, severe complications (e.g. secondary infection, cyst infection and hydrops) may result from leakage of $\mathrm{CE}$ cysts [4]. Albendazole (ABZ), as a chemotherapeutic agent used for the treatment of CE, has been commonly utilized in clinical practice, but its efficiency is hampered due to its disadvantages including poor absorption in gastrointestinal tract, poor solubility in water and organic solvents, as well as low concentrations in the parasite cysts [5]. Moreover, poor response was reported after medication of patients with hydatid cysts in the liver and lung, particularly those with bone hydatid disease. Therefore, it is necessary to develop new methods for the treatment of $\mathrm{CE}$. 
Radiotherapy may represent an alternative treatment modality for $\mathrm{CE}$ as it may induce cellular apoptosis or necrosis of $\mathrm{CE}$, but up to now, there is no adequate evidence for it [6]. Only a few studies have been reported on the efficiency of radiotherapy in treating $\mathrm{CE}$ and the use of radiotherapy for the treatment of $\mathrm{CE}$ is still controversially discussed [7-9]. Besides, there are still disputes on the efficiency of radiotherapy on the treatment of CE infection. For example, in 1998, Schimid reported decrease of cyst in size after treatment using gamma knife radiosurgery in a case with cerebral alveolar hydatid disease [7]. In 2013, Ulger et al. revealed stable disease and absence of chest pain in one case who had failed the surgical procedures and medical treatment at the 1-year follow up after radiotherapy [8]. On the contrary, Pohle et al. concluded that radiotherapy should not be recommended for the management of alveolar echinococcosis as it had no clear-cut parasiticidal effects [9]. In 2013, radiotherapy using heavy ions was reported to promote the apoptosis of hydatid cysts through modulation of Caspase 3 expression [10]. However, radiotherapy using heavy ions has been rarely performed in hospitals due to technical limitations. In this study, we investigated the parasiticidal effects of X-ray on the metacestodes of E. granulosus that mimicked the activities of $E$. granulosus in vivo. In addition, potential mechanisms were investigated.

\section{Methods}

\section{Study setting and design}

Fresh sheep liver samples infected with E. granulosus were used in the study. The sheep were obtained from a local sheep nursery in Xinjiang Autonomous Region. The animals were sacrificed according to the guide for the care and use of laboratory animals (8th edition). The study protocols were in line with the guidelines proposed by the First Affiliated Hospital of Xinjiang Medical University. Protoscoleces were subjected to irradiation, or they were cultured in vitro to investigate the effects of $\mathrm{X}$ ray on the encystation.

\section{Culture of protoscoleces}

The liver surface was washed with water, followed by sterilization using 75\% ethanol. The hydatid fluid containing protoscoleces were obtained under aseptic conditions. Protoscoleces were washed using PBS ( $\mathrm{pH}$ 7.2) for three times, followed by staining with $0.1 \%$ eosin for 5 min as previously described [11]. Then the staining results were observed under a light microscope. Dead protoscoleces stained in red color, and exhibited structural damages. Protoscoleces with a survival rate of $>90 \%$ were used for the subsequent culture. The remaining protoscoleces were transferred to RPMI 1640 medium (HyClon, USA) containing 10\% fetal bovine serum (HyClon, USA), and cultivated at $37{ }^{\circ} \mathrm{C}$ in $5 \% \mathrm{CO}_{2}$. Encystation was noticed on day 10, and intact E. granulosus cyst were formed between day 14 and day 20 .

\section{Effects of X-ray on the encystation of protoscoleces}

Protoscoleces with a survival rate of $>90 \%$ were divided into control group and X-ray groups subjected to X-ray with an intensity of $10 \mathrm{~Gy}, 20 \mathrm{~Gy}, 30 \mathrm{~Gy}, 40 \mathrm{~Gy}, 50 \mathrm{~Gy}$, and 60 Gy [12], respectively. The viability was qualitatively determined using the eosin staining (0.1\%) for 5 min, followed by observation using a microscope. The encystation rate was calculated based on the ratio of encystation to the total protoscoleces. The criteria of encystation were as follows: head enlargement, somites increase, integration of somites and hooklets, degradation of calcium granules, absence of rostellum [13]. The survival rate was calculated according to the number of protoscoleces underwent necrosis (staining in red color) in 100 protoscoleces. After cultivation for 14 days, the number of protoscoleces with encystation was observed under a microscope. The ratio of encystation was presented as the percentage of protoscoleces with encystation to the total number of protoscoleces.

\section{Effects of X-ray on the CE encystation}

$\mathrm{CE}$ cysts were divided into four groups, including control group subject to no $\mathrm{X}$-ray or $\mathrm{X}$-ray groups subject to X-ray with an intensity of $30 \mathrm{~Gy}, 45 \mathrm{~Gy}$ and $60 \mathrm{~Gy}$, respectively. In each group, three culture flasks containing a total of 600 cysts (200 cysts in each culture flask) were subjected to an irradiation dosage of $300 \mathrm{cGy} / \mathrm{min}$ at least three times. The distance between the X-ray source and the cyst was $100 \mathrm{~cm}$. The irradiation field was $10 \times 10 \mathrm{~cm}$. The irradiation intensity was selected according to a dose escalation regimen, and the maximal intensity was selected to be 60 Gy that had been confirmed to be safety in human beings. The time interval of the 3 radiation cycle was 2 days, and was accomplished within 1 week. The radiation cycle and irradiation intensity were established according to the frequency and dosage of regimen used for treating liver cancer in clinical practice.

\section{Microscopic observation}

On day 7, 100 cysts obtained from each group were observed under a light microscope to observe the morphology of the cysts. The protoscoleces were put onto slides, covered with coverslips, and observed microscopically under various magnifications.

\section{Transmission electron microscopy (TEM)}

Protoscoleces were rinsed once with saline, fixed in $4 \%$ glutaraldehyde $(24 \mathrm{~h})$ and $1 \%$ osmium tetroxide sequentially, dehydrated with acetone gradient, and embedded 
in Epon 812 epoxy resin. The sections $(60 \mathrm{~nm})$ were cut with an ultrathin section machine, stained with uranium and lead electron stains, and observed under the TEM (JEOL1230, Japan).

\section{Real-time PCR}

Total RNA was extracted from the cysts using TRIzol according to manufacturer's instructions (Invitrogen, USA). The cDNA synthesis was carried out with approximately $2 \mu \mathrm{g}$ RNA using the RevertAid First Strand cDNA Synthesis Kit (Thermo Fisher, USA). Real-time PCR was conducted using SYBR Green (QiaGen, Germany) on a Bio-Rad 785BR10642 system (Singapore) with the primers listed in Table 1, according to the manufacturer's instructions. The PCR amplifications were as follows: $95{ }^{\circ} \mathrm{C}$ for 14 min, followed by 35 cycles of $95^{\circ} \mathrm{C} 20 \mathrm{~s}$, various annealing temperatures (EgEPC1: $55{ }^{\circ} \mathrm{C}$; Caspase-3: $53{ }^{\circ} \mathrm{C}$; EgTPX: $56{ }^{\circ} \mathrm{C}$; EgHSP70: $\left.56{ }^{\circ} \mathrm{C}\right) 30 \mathrm{~s}$, and $72{ }^{\circ} \mathrm{C} 30 \mathrm{~s}$, as well as $65^{\circ} \mathrm{C}$ for $10 \mathrm{~min}$. The mRNA level was normalized by beta-actin. The amplification results for real-time PCR was calculated as $2^{(-\Delta \Delta \mathrm{Ct})}$, according to the previous description [14].

\section{Statistical analysis}

Data were analyzed by SPSS 17 0. Each test was performed at least in triplicate. Analysis of variance (ANOVA) was used for inter-group comparison. $P<0.05$ was considered statistically significant.

\section{Results}

\section{Effects of X-ray exposure on the encystation rate of} protoscoleces

On day 14, encystation was noticed in the majority of protoscoleces in the control group. Meanwhile, presence of the corneum layer was noticed in the peripheral part of the cyst. In the X-ray groups, the encystation rate was significantly decreased compared to the control group $(P<0.05)$, especially the groups subjected to a dose of 40 Gy or more $(P<0.01$, Fig. 1$)$. Radiation with an intensity of 60 Gy could completely inhibit the encystation.

Table 1 Specific primers of EgTPX, EgEPC1 and EgHSP7O

\begin{tabular}{lll}
\hline Gene & Primers (5'-3') & Product size \\
\hline EgTPX & F: TTCTTAGATAAGCTCGACTCCAA & $196 \mathrm{bp}$ \\
& R: AGTATATAGACCGGGAATAAGGG & \\
EgHSP70 & F: GAGGAGTGTGTTCGACCT & $145 \mathrm{bp}$ \\
& R: GTCCGGGTTATCGACTTG & \\
EgEPC1 & F: TITTTAGATAAGCTCGACTCCAA & $151 \mathrm{bp}$ \\
& R: AGTATATAGACCGGGAATAAGGG & \\
Actin & F: TCAATCCTAAAGCCAATC & $163 \mathrm{bp}$ \\
& R: CGTACAACGACAGCAC & \\
\hline
\end{tabular}

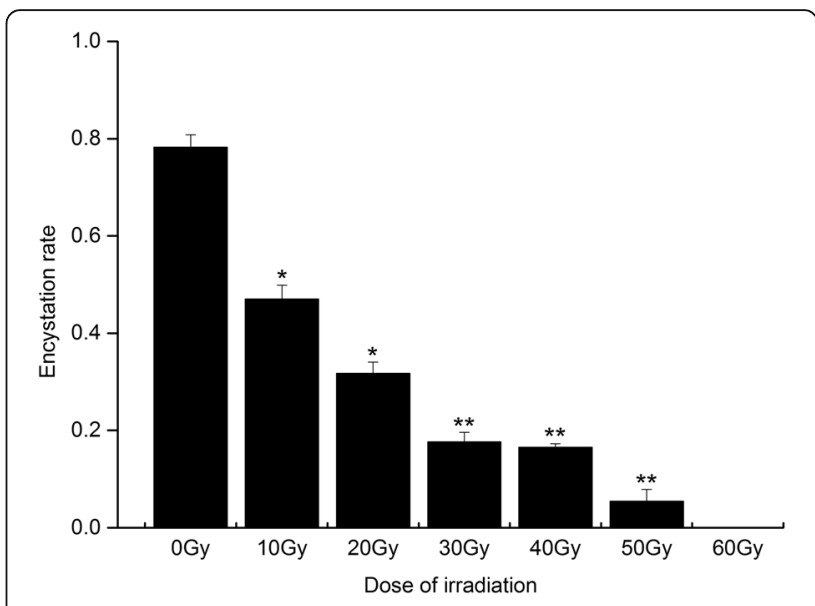

Fig. 1 Encystation rate of protoscoleces after irradiation. ${ }^{*} P<0.05$ versus control group; ${ }^{* *} P<0.01$ versus control group

\section{Morphology of protoscoleces after irradiation}

Compared with the control group, the structure of the protoscoleces was interrupted after X-ray exposure. In the control group, the protoscoleces were well developed with a higher encystation rate. The corneum layer could be observed around the cyst. The arrangement of hooklets on the rostellum was regular. In contrast, in the irradiation group, the hooklets on the rostellum were deranged, and malformation was noticed in the suckers in a dose dependent manner (Fig. 2).

\section{X-ray induced cystic wall atrophy}

In this part, we determined the growth of hydatids 1 week after X-ray exposure using light microscope. In the control group, the cyst wall was intact (Fig. 3a). On the contrary, in the X-ray groups, atrophy was noticed in the cyst walls. In addition, the structure of the cyst was completely disrupted after X-ray exposure (Fig. 3b-d).

\section{TEM findings}

In the control group, the arrangement of the hydatid cysts was regular. The morphologies of cortex cells were intact. The nuclear structure was tight, and the nucleolus was clear. Meanwhile, electron dense bodies were dispersed in the cysts. However, after X-ray exposure, the structures were interrupted, together with dilatation of endoplasmic reticulum. Necrosis was noticed in the cortical cells, together with broken nuclei and disappearance of nucleoli (Fig. 4b). In addition, the cortical area was thin, and the microvilli were decreased in number (Fig. 4c). Particularly, in the 60 Gy group, swelling of mitochondria was noticed in the mitochondria in the cortical cells, combined with increase of lysosome and formation of myeloid body (Fig. 4d). 


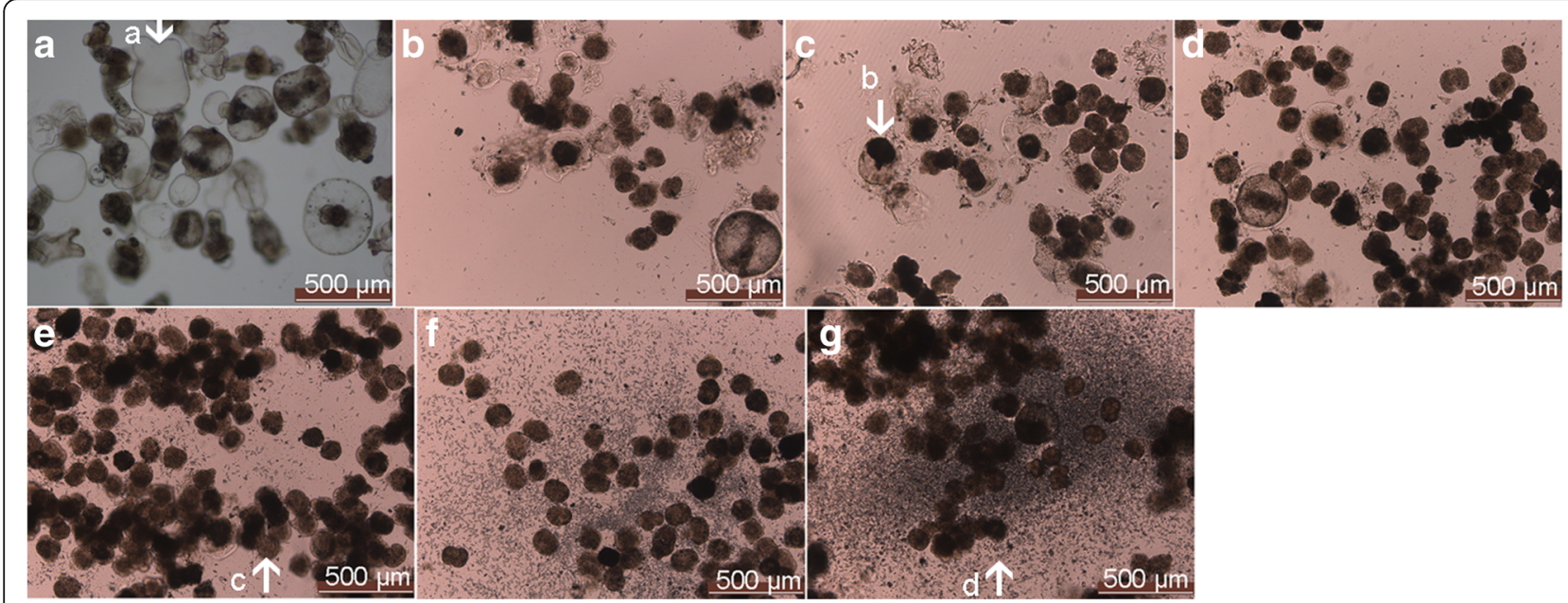

Fig. 2 Morphologies of protoscoleces on day 14 after irradiation with a intensity of 0 Gy (a), 10 Gy (b), 20 Gy (c), 30 Gy (d), 40 Gy (e), 50 Gy (f), and $60 \mathrm{~Gy}(\mathbf{g})$. The protoscoleces were well developed in the control group, while interruption of hooklets was noticed in the irradiation group in a dose dependent manner. The images were observed under a magnification of 100x. a: normally developed and motile protoscoleces; b: non-motile protoscoleces; c: protoscoleces subject to necrosis; d: breakdown hooks

\section{Expression of EgTPX, EgHSP70, EgEPC1 and Caspase-3 in} cyst after irradiation

The expression of EgTPX was down-regulated in the cyst after irradiation with a dose of 15 Gy and 30 Gy compared with the normal control. However, significant increase was noticed in the expression of $E g T P X$ at a dose of 45 Gy and 60 Gy (Fig. 5a). The expression of EgHSP70 was down-regulated after irradiation with a dose of 15 Gy compared with the control group. Nevertheless, its expression was significantly up-regulated after irradiation with a dose of $30 \mathrm{~Gy}, 45 \mathrm{~Gy}$ and
60 Gy compared with the control (Fig. 5b). For the expression of EgEPC1 and Caspase-3, a remarkable down-regulation was noticed after a dose of 15 Gy or 30 Gy compared with the control group, but the expression was significantly up-regulated after a dose of 45 Gy or 60 Gy, respectively (Fig. 5c and d).

\section{Discussion}

Previously, extensive studies have been carried out to investigate the efficiency of radiotherapy on $E$. granulosus protoscoleces $[15,16]$. However, few studies have been

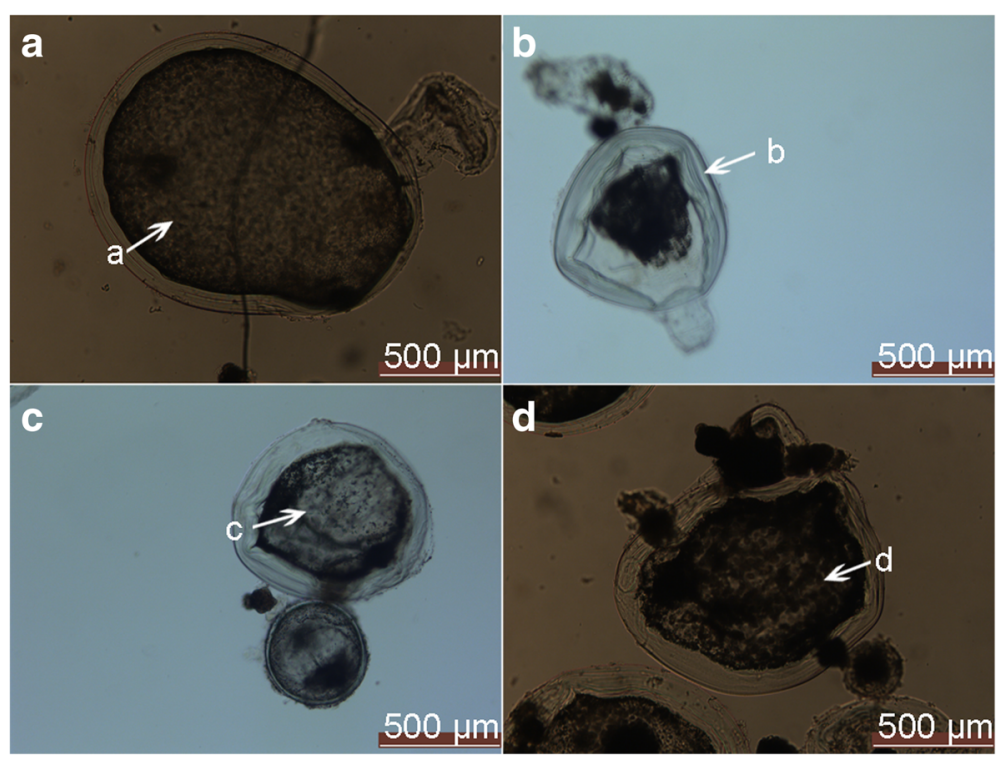

Fig. 3 Morphologies of cyst after irradiation with a intensity of 0 Gy (a), 30 Gy (b), 45 Gy (c), and 60 Gy (d). The images were observed on day 7 under a magnification of 100x. a: calcium granules; b: collapsed cyst wall; c: elimination of calcium granules; d: cellular debris 


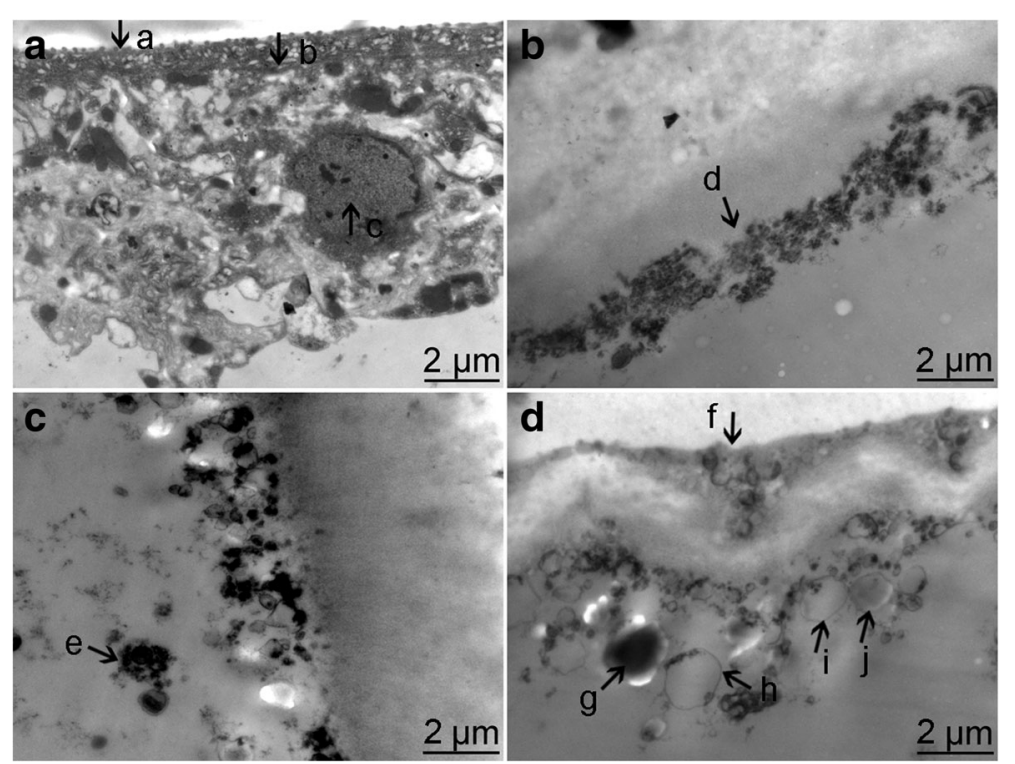

Fig. 4 Morphology of cyst after irradiation under TEM. a The cystic structure was normal in the normal control. b-d: Cystic structures after irradiation under a density of $30 \mathrm{~Gy}, 45 \mathrm{~Gy}$ and $60 \mathrm{~Gy}$. a: microtriches; b: tegument; c: undifferentiated cells; d. broken germinal layer; e: aberrantly condensed chromosomal masses; f: broken tegument; g: lipid droplets; h-j: empty vesicles

carried out to investigate the efficiency of irradiation on metacestodes $[9,10]$. In this study, we reported the effects of X-ray on in vitro cultured E. granulosus protoscoleces and the development of metacestodes, as well as already formed metacestodes. In addition, our study showed that irradiation showed parasiticidal effects through promoting the apoptosis in vitro.

Radiotherapy has been commonly used for the management of patients with cancer. Indeed, there are some side effects for radiotherapy such as percutaneous reaction,
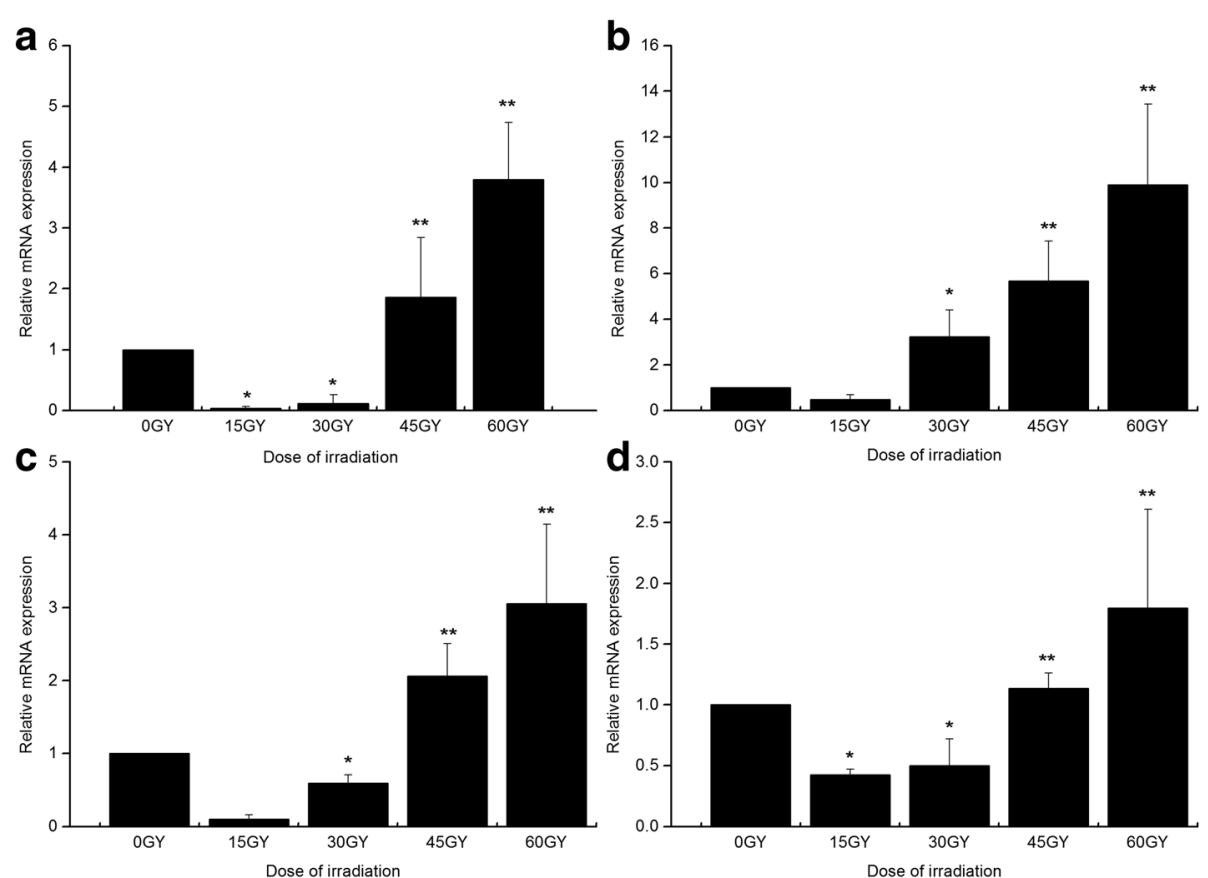

Fig. 5 Expression of EgTPX (a), EgHSP7O (b), EgEPC1 (c) and Caspase-3 (d) in cyst after irradiation in hydatid cysts. Gene expression was determined using Real-Time PCR. All the tests were performed at least in triplicate. ${ }^{*} P<0.05$ versus control group; ${ }^{* *} P<0.01$ versus control group 
mucous reaction and bone marrow depression [17]. In this study, the irradiation dose was selected based on the tolerance of the tissues, in order to provide the rational for the potential application in vivo in future studies. To our best knowledge, only a few studies have been carried out to investigate the efficiency of radiotherapy on the management of CE [6]. Previously, Schmid reported a case of cerebral alveolar hydatid disease showed decrease of cyst diameter after treating with gamma knife radiosurgery and albendazole. The patient was followed up for at least 3 years with no nervous system symptoms [7]. In 2003, Park et al. investigated the survival of metacercariae irradiated with various amounts of gamma knife (5 Gy - 50 Gy) [18]. Compared with those of non-irradiated controls, recovery rates of adult worms in irradiated groups were reduced gradually with increased irradiation doses. No worm was recovered from rats fed with 50 Gy irradiated metacercariae. In this study, the structure of the protoscoleces was interrupted after X-ray exposure compared with the control group. To confirm the parasiticidal effects of $\mathrm{X}$-ray on cysts, the cultured cysts in vitro were subject to irradiation, and the changes of the structures were observed using light microscope and TEM, respectively. Light microscope findings indicated presence of atrophy and collapse in the cyst wall, while in the control group, the wall was intact with no interruption. TEM showed dilatation of endoplasmic reticulum and aberrant condensed mass, which represented structural damages after irradiation, however, in the control group, the cysts were regularly arranged. On this basis, X-ray could interrupt the structure of hydatid cysts and induce apoptosis or necrosis.

Few studies have been carried out to investigate the molecular mechanism of irradiation on the hydatid cysts, and most of the studies have focused on the apoptosis [11]. For example, in a previous study, Yuan et al. revealed gamma knife induced higher mortality and up-regulation of Caspase-3 in the protoscoleces [19]. Besides, Zhou et al. reported that heavy ions could promote the apoptosis of hydatid cysts through modulation of the expression of Caspase 3 [10]. In order to investigate the potential mechanism in the irradiation on the hydatid cysts, we determined the expression of 3 genes that have been reported to be closely related to the growth of hydatids (i.e. EgTPX, EgEPC1 and EgHSP70) and the caspase gene related to cellular apoptosis. The EgTPX gene was reported to involve in the clearance of reactive oxygen [20]. In this study, our results indicated that the expression of EgTPX was decreased with the increase of irradiation between 15 Gy and 30 Gy. On the contrary, its expression was increased with the increase of irradiation dose between $45 \mathrm{~Gy}$ and $60 \mathrm{~Gy}$. On this basis, it is reasonable to conclude that a dose of 15-30 Gy inhibited the clearance capacity of hydatids to the oxygen radicals, while a dose of
45-60 Gy contributed to the clearance of oxygen radicals. For the expression of EgHSP70 with anti-oxidant capacities closely involved in the protein translation [21], transfer and degradation, our results showed it was obviously increased upon irradiation with a dose of 30 Gy or more. With regards to the expression of Caspase-3, Hu et al. revealed Caspase 3 involved in the apoptosis of protoscolex cells [11]. In this study, the expression of Caspase 3 was decreased in a dose of 15-30 Gy, while it increased upon irradiation of a dose of 45-60 Gy. This indicated that a dose of 45-60 could trigger the apoptosis. Interestingly, as an epitope gene of E. granulosa, the expression of $E g E P C 1$ was down-regulated in presence of irradiation of $45 \mathrm{~Gy}$ and $60 \mathrm{~Gy}$. In future, further studies are needed to investigate the potential causes.

Generally, hydatid cyst is considered as the life origin of E. granulosa. To our best knowledge, the research and management of $E$. granulosa related infection is hampered due to lacking of in vitro cultivation of metacestodes. In this study, we successfully cultivated metacestodes in vitro from the protoscoleces obtained from naturally infected sheep. On this basis, for the first time, we investigated the killing effects of irradiation on the metacestodes.

\section{Conclusion}

We firstly investigated the parasiticidal effects of X-ray on the metacestodes of E. granulosa in vitro. X-ray showed parasiticidal effects and could modulate the phenotype and gene expression of the metacestodes. For the mechanism, it may be associated with the interruption of structure and cellular apoptosis.

\section{Acknowledgements}

Not applicable

Funding

This study was supported by the Natural Science Foundation of Xinjiang Uygur Autonomous Region (No. 2016D01C324).

\section{Availability of data and materials}

The datasets during and/or analyzed during the current study available from the corresponding author on reasonable request.

\section{Authors' contributions}

MR, WG wrote the manuscript. WH, LPF and LJ did the experiments. AA, BYW SMY did the data analysis. BYX, ZWB revised the manuscript. All authors read and approved the final manuscript.

Ethics approval and consent to participate

The First Affiliated Hospital of Xinjiang Medical University approved the animal experiments.

\section{Consent for publication}

Not applicable

\section{Competing interests}

The authors declare that they have no competing interests.

\section{Publisher's Note}

Springer Nature remains neutral with regard to jurisdictional claims in published maps and institutional affiliations. 
Received: 31 October 2016 Accepted: 15 September 2017

\section{Published online: 21 September 2017}

\section{References}

1. Brunetti $E$, Kern P, Vuitton DA. Expert consensus for the diagnosis and treatment of cystic and alveolar echinococcosis in humans. Acta Trop. 2010;114(1):1-16.

2. McManus DP, Thompson RCA. Molecular epidemiology of cystic echinococcosis. Parasitology. 2003;127(S1):S37-51.

3. Sarkar S, Roy H, Saha P, Sengupta M, Sarder K, Sengupta M. Cystic echinococcosis: a neglected disease at usual and unusual locations. Trop Parasitol. 2017;7(1):51-5.

4. Li Y, Zheng H, Cao X, Liu Z, Chen L. Demographic and clinical characteristics of patients with anaphylactic shock after surgery for cystic echinococcosis. Am J Trop Med Hyg. 2011;85(3):452-5.

5. Nazligul $Y$, Kucukazman M, Akbulut $S$. Role of chemotherapeutic agents in the management of cystic echinococcosis. Int Surg. 2015;100(1):112-4.

6. Xie Z, Chen L, Xie Q, et al. Surgery or radiotherapy for the treatment of bone hydatid disease: a retrospective case series. Int J Infect Dis. 2015;33:114-9.

7. Schmid M, Pendl G, Samonigg H, Ranner G, Eustacchio S, Reisinger EC Gamma knife radiosurgery and albendazole for cerebral alveolar hydatid disease. Clin Infect Dis. 1998;26(6):1379-82.

8. Ulger $\mathrm{S}$, Barut $\mathrm{H}$, Tunc $\mathrm{M}$, et al. Radiation therapy for resistant sternal hydatid disease. Strahlenther Onkol. 2013;189(6):508-9.

9. Pohle S, Ernst R, MacKenzie C, et al. Echinococcus multilocularis: the impact of ionizing radiation on metacestodes. Exp Parasitol. 2011;127(1):127-34.

10. Zhou $X$, Zhao $Y$, Zhou R, Zhang $H$. Suppression of E. multilocularis hydatid cysts after ionizing radiation exposure. PLoS Negl Trop Dis. 2013;7(10):e2518.

11. Hu H, Kang J, Chen R, Mamuti W, Wu G, Yuan W. Drug-induced apoptosis of Echinococcus granulosus protoscoleces. Parasitol Res. 2011;109(2):453-9.

12. Sawrie SM, Fiveash JB, Caudell JJ. Stereotactic body radiation therapy for liver metastases and primary hepatocellular carcinoma: normal tissue tolerances and toxicity. Cancer Control. 2010;17(2):111-9.

13. Zhang W, Macolm K, Li J, McManus DP. Echinococcus granulosus: pre-cultue of protoscoleces in vitro significantly increases development and viability of secondary hydatid cysts in mice. Exp Parasitol. 2005;110:88-90.

14. Yuan JS, Reed A, Chen F, Stewart CN. Statistical analysis of real-time PCR data. BMC Bioinformatics. 2006;7(1):1.

15. Bao YX, Zhang YF, Ni YQ, et al. Effect of $6 \mathrm{MeV}$ radiotherapy on secondary Echinococcus multilocularis infection in rats. Zhongguo Ji Sheng Chong Xue Yu Ji Sheng Chong Bing Za Zhi. 2011;29(2):127-9.

16. Cornejo-Juárez P, Espinosa-Altamirano A, Ibarra-del-Río M, Pacheco-Bravo I, Volkow-Fernández P. Cystic echinococcosis: a disease mimicking cancer in a non-endemic country report of two cases. West Indian Med J. 2013;62(3):266-9.

17. Bentzen SM. Preventing or reducing late side effects of radiation therapy: radiobiology meets molecular pathology. Nat Rev Cancer. 2006;6(9):702-13.

18. Park G-M, Yong T-S. Effects of gamma-irradiation on the infectivity and chromosome aberration of Clonorchis Sinensis. Korean J Parasitol. 2003:41(1):41-5

19. Yuan QLB, Hu K, Zhang MY, Duo J, Chen C, Li X, Xiang ZW. Killing effect on in vitro exposure of Echinococcus protoscoleces to gamma-ray. Zhongguo Ren Shou Gonghuanbing Xuebao. 2013;29:904-8.

20. Wang H, Hou Q-I, Zhang Z-Z, Zhang F-c, Zhang W-b. Cloning, fusion expression and identification of gene encoding the thioredoxin peroxidase from Echinococcus granulosus. Chin J Zoonoses. 2008;5:019.

21. Ortona $E$, Margutti $P$, Delunardo $F$, et al. Molecular and immunological characterization of the C-terminal region of a new Echinococcus granulosus heat shock protein 70. Parasite Immunol. 2003:25(3):119-26.

\section{Submit your next manuscript to BioMed Central and we will help you at every step:}

- We accept pre-submission inquiries

- Our selector tool helps you to find the most relevant journal

- We provide round the clock customer support

- Convenient online submission

- Thorough peer review

- Inclusion in PubMed and all major indexing services

- Maximum visibility for your research

Submit your manuscript at www.biomedcentral.com/submit
C Biomed Central 